\title{
CALIBRATION OF CENTRAL CATADIOPTRIC CAMERA WITH ONE-DIMENSIONAL OBJECT UNDERTAKING GENERAL MOTIONS
}

\author{
Xiaoming Deng ${ }^{1}$, Fuchao $W^{2}$, Yihong $W^{2}$, Liang Chang ${ }^{3}$, Wei Liu ${ }^{1}$, Hongan Wang ${ }^{1}$ \\ ${ }^{1}$ State Key Laboratory of Computer Science, Institute of Software,Chinese Academy of Sciences \\ ${ }^{2}$ National Laboratory of Pattern Recognition, Institute of Automation, Chinese Academy of Sciences \\ ${ }^{3}$ College of Information Science and Technology, Beijing Normal University \\ E-mails: $\{$ idengxm,hongan.w $\} @$ gmail.com, $\{$ fcwu,yhwu $\} @$ nlpr.ia.ac.cn
}

\begin{abstract}
A $1 \mathrm{D}$ object is a segment with several known-distance markers, and calibration methods with $1 \mathrm{D}$ objects are more flexible than those with $2 \mathrm{D} / 3 \mathrm{D}$ objects. Under the pinhole camera model, it is proved that the calibration with free-moving $1 \mathrm{D}$ objects is not possible. For a central catadioptric camera setup, can the camera be calibrated by a $1 \mathrm{D}$ object under general motions? In this paper, we prove that a central catadioptric camera can indeed be calibrated, and propose a catadioptric camera calibration method using 1D objects undertaking general motions. The proposed method consists of two steps. Firstly, the principal point is calculated with geometric invariants under catadioptric camera model; Secondly, we use images of $1 \mathrm{D}$ object to calibrate the focal lengths, skew factor and mirror parameter. The method needs neither prior knowledge of catadioptric parameters nor conic fitting, and it is linear, which makes it easy to implement. Experiments demonstrate its usefulness and stability.
\end{abstract}

Index Terms - Camera calibration, 1D object

\section{INTRODUCTION}

Central catadioptric cameras are widely used in many applications such as visual surveillance, virtual reality and robotics[1][2][3]. Calibration of central catadioptric camera is of great importance for applications, however it is challenging due to the heavy distortion in the catadioptric images. This paper aims to propose a linear and flexible calibration method for central catadioptric cameras.

Representative studies on the calibration of catadioptric cameras have appeared in recent years, and they can be summarized into two categories by the type of calibration objects[4] [5][6]. (1) Point based calibration. Aliaga[7] proposed 3D control points based calibration method. The intrinsic and extrinsic parameters can be obtained by a nonlinear method with 3D points. Since the image formation of central catadioptric camera is nonlinear, point-based calibration methods employ nonlinear optimization method and requires a good initial estimation to start the minimization, which is prone to reach local minimizations. (2) Line based calibration. Barreto and Araujo [8] studied the geometric properties of line images under the central catadioptric model, and proved that the central catadioptric camera with a hyperbolic/elliptical mirror can be calibrated from two line images and that with a parabolic mirror can be calibrated from at least three line images. Most of line-based calibrations for a central catadioptric camera need fittings of partial visible conics(about one-third of a conics), a hard problem, and the camera intrinsic parameters in turn cannot be accurately estimated.

A 1D calibration object is a segment with several known-distance points, and calibration methods with 1D objects are more flexible than those with 2D or 3D objects[9][10]. Under the pinhole camera model,
Zhang[9] proved that the calibration is not possible with free-moving $1 \mathrm{D}$ object, but can be done only if one of the markers is fixed. In our paper, we propose a catadioptric camera calibration method using 1D objects with four or more known points. Our method can calibrate a catadioptric camera with the 1D object undertaking five or more general motions. The principal point is calculated with geometric invariants under catadioptric camera model, and we use images of 1D object to calibrate the focal lengths, skew factor and mirror parameter. Our calibration method with $1 \mathrm{D}$ objects has the following advantages:

1. No fitting of image points to conics is required. The principal point can be calculated linearly with geometric invariants under catadioptric camera, and three image points from the same $1 \mathrm{D}$ object can directly give a linear equation on the other catadioptric parameters. This is a difference compared with the previous methods using lines, in which conic fitting for line images are required. As it is known for catadioptric cameras only small portion of conics of line images is visible, and the usual conic fitting methods do not work well[4][8].

2. No prior knowledge to the catadioptric parameters is required. In literatures, the visible imaged mirror contour, the field of view and the mirror parameter $\xi$ are often used for calibrating a central catadioptric system.

\section{CENTRAL CATADIOPTRIC CAMERA}

Geyer and Daniilidis [11] proposed a generalized image formation model for central catadioptric camera as shown in Fig. 1(a). A 3D point $\mathbf{X}$ is projected to a point $\mathbf{X}^{s}$ on a unit sphere centered at the viewpoint $\mathbf{O}$, then projected to a point $\mathbf{m}=(u, v, 1)^{T}$ on the image plane $\Pi$ by a pinhole camera through the perspective center $\mathbf{O}^{c}$. The image plane is perpendicular to the line defined by the viewpoints $\mathbf{O}$ and $\mathbf{O}^{c}$. The image formation process can be explicitly expressed as follows[4]:

$$
\begin{gathered}
\lambda \mathbf{m}=\mathbf{K}\left(\frac{\mathbf{R X}+\mathbf{t}}{\|\mathbf{R X}+\mathbf{t}\|}+(0,0, \xi)^{T}\right), \mathbf{K}=\left(\begin{array}{lll}
r f & s & u_{0} \\
0 & f & v_{0} \\
0 & 0 & 1
\end{array}\right) \\
\lambda=\frac{\xi+\sqrt{\xi^{2}+\left(1-\xi^{2}\right) \mathbf{m}^{T} \mathbf{K}^{-T} \mathbf{K}^{-1} \mathbf{m}}}{\mathbf{m}^{T} \mathbf{K}^{-T} \mathbf{K}^{-1} \mathbf{m}}
\end{gathered}
$$

where $\lambda$ is the projective depth of $\mathbf{X}^{s}$ under the pinhole camera, $\mathbf{R}$ is the rotation matrix and $\mathbf{t}$ the translation vector, $\mathbf{K}$ the camera intrinsic matrix, with $f$ the focal length, $r$ the aspect ratio and $s$ the skew factor. The vector $\mathbf{p}=\left(u_{0}, v_{0}, 1\right)^{T}$ is the homogenous coordinate 


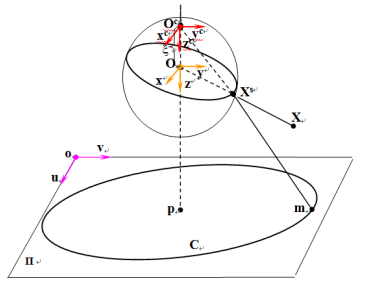

(a)

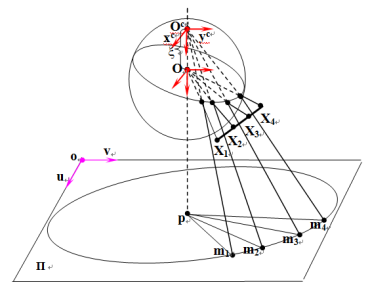

(b)
Fig. 1. (a) Image formation of the central catadioptric camera. The image points of the $1 \mathrm{D}$ calibration object.

of principal point, $\xi$ is often called as the mirror parameter, which determines the type of mirror in the central catadioptric camera.

For calibrating catadioptric cameras, there are totally six parameters $\left\{f, r, s, u_{0}, v_{0}, \xi\right\}$ to be determined, and the parameters are also called as catadioptric parameter in this paper.

\section{CALIBRATION ALGORITHM}

\subsection{Principal Point}

Let $\boldsymbol{\Pi}_{j}$ be the planes defined by the optical axis $\mathbf{O O}^{c}$ and the point $\mathbf{X}_{j}$ on the 1D calibration object, and $\mathbf{p}=\left(u_{0}, v_{0}, 1\right)^{T}$ be the principal point of the camera, i.e. the intersection of the optical axis $\mathbf{O O}^{c}$ with the image plane $\Pi$ (See Fig.1(b)).

Each pair of $\mathbf{X}_{i}$ and its image $\mathbf{m}_{i}=\left(u_{i}, v_{i}, 1\right)^{T}$ are coplanar with the optical axis $\mathbf{O O}_{c}$ (See Fig.1(b)), and thus the intersections of the pencil of planes $\mathbf{O O}_{c}\left(\mathbf{X}_{1}, \mathbf{X}_{2}, \mathbf{X}_{3}, \mathbf{X}_{4}\right)$ with the image plane is the pencil of lines $\mathbf{p}\left(\mathbf{m}_{1}, \mathbf{m}_{2}, \mathbf{m}_{3}, \mathbf{m}_{4}\right)$, so the cross ratio of $\mathbf{p}\left(\mathbf{m}_{1}, \mathbf{m}_{2}, \mathbf{m}_{3}, \mathbf{m}_{4}\right)$ is equal to the cross ratio of $\mathbf{O O}_{c}\left(\mathbf{X}_{1}, \mathbf{X}_{2}, \mathbf{X}_{3}, \mathbf{X}_{4}\right)$ and the cross ratio of pencil of lines $\mathbf{O}\left(\mathbf{X}_{1}, \mathbf{X}_{2}, \mathbf{X}_{3}, \mathbf{X}_{4}\right)$ similarly, which is equal to the cross ratio of $\left\{\mathbf{X}_{1}, \mathbf{X}_{2}, \mathbf{X}_{3}, \mathbf{X}_{4}\right\}$ because $\left\{\mathbf{X}_{1}, \mathbf{X}_{2}, \mathbf{X}_{3}, \mathbf{X}_{4}\right\}$ are collinear[12]. Therefore, we have

$$
\operatorname{Cross}\left(\mathbf{X}_{1}, \mathbf{X}_{2} ; \mathbf{X}_{3}, \mathbf{X}_{4}\right)=\frac{\left|\mathbf{p m}_{1} \mathbf{m}_{3}\right| \cdot\left|\mathbf{p m}_{2} \mathbf{m}_{4}\right|}{\left|\mathbf{p m}_{1} \mathbf{m}_{4}\right| \cdot\left|\mathbf{p} \mathbf{m}_{2} \mathbf{m}_{3}\right|}
$$

where $\operatorname{Cross}(\cdot)$ is the cross ratio of four collinear points, and $\frac{\left|\mathbf{p m}_{1} \mathbf{m}_{3}\right| \cdot\left|\mathbf{p m}_{2} \mathbf{m}_{4}\right|}{\left|\mathbf{p} \mathbf{m}_{1} \mathbf{m}_{4}\right| \cdot\left|\mathbf{p m}_{2} \mathbf{m}_{3}\right|}$ is the cross ratio of $\mathbf{p}\left(\mathbf{m}_{1}, \mathbf{m}_{2}, \mathbf{m}_{3}, \mathbf{m}_{4}\right)$, and |. $\mid$ stands for a determinant of a matrix.

Let $r_{1234}=\operatorname{Cross}\left(\mathbf{X}_{1}, \mathbf{X}_{2} ; \mathbf{X}_{3}, \mathbf{X}_{4}\right)$. Since the distances between any two points in $\left\{\mathbf{X}_{1}, \mathbf{X}_{2}, \mathbf{X}_{3}, \mathbf{X}_{4}\right\}$ are known, we can compute the cross ratio, and then we have the following quadric constraints on the principal point $\mathbf{p}$ by equation (3)

$$
\left|\mathbf{p m}_{1} \mathbf{m}_{3}\right| \cdot\left|\mathbf{p m}_{2} \mathbf{m}_{4}\right|=r_{1234}\left|\mathbf{p m}_{1} \mathbf{m}_{4}\right| \cdot\left|\mathbf{p m}_{2} \mathbf{m}_{3}\right|
$$

Generally speaking, equation (4) is a homogenous equation about $u_{0}^{2}, u_{0} v_{0}, v_{0}^{2}, u_{0}, v_{0}, 1$. If at least five independent constraints are available, we can regard $u_{0}^{2}, u_{0} v_{0}, v_{0}^{2}, u_{0}, v_{0}, 1$ as independent variables, and then the principal point $\mathbf{p}=\left(u_{0}, v_{0}, 1\right)^{T}$ can be solved linearly by singular value decomposition. In order to uniquely determine the principal point, we need at least five images of the 1D calibration object with different space positions.

If $1 \mathrm{D}$ calibration object and the optical axis are coplanar, then the equations (4) become identical equations. However, from the collinearity we can obtain linear constraints on the principal point, $\left|\mathbf{p m}_{i} \mathbf{m}_{j}\right|=0(1 \leq i<j \leq 4)$, in which only one independent constraint about $\mathbf{p}$ is enforced. Therefore, we can determine principal point by at least two images of the 1D object at different positions.

After the principal point $\mathbf{p}$ is calibrated, the origin of image coordinate system can be translated to $\mathbf{p}$ by a linear transformation:

$$
\mathbf{T}_{p}=\left(\begin{array}{ccc}
1 & 0 & -u_{0} \\
0 & 1 & -v_{0} \\
0 & 0 & 1
\end{array}\right)
$$

Hence, the point $\mathbf{m}$ can be translated to $\tilde{\mathbf{m}}$ by $\tilde{\mathbf{m}}=\mathbf{T}_{p} \mathbf{m}=$ $\left(u-u_{0}, v-v_{0}, 1\right)^{T}$, and $\mathbf{p}$ is translated to $\tilde{\mathbf{p}}=(0,0,1)^{T}$. Hereafter, we denote $u \triangleq u-u_{0}, v \triangleq v-v_{0}, \mathbf{m} \triangleq \tilde{\mathbf{m}}$ and $\mathbf{p} \triangleq \tilde{\mathbf{p}}$ for simplicity.

Denote $\tilde{\mathbf{K}}$ as

$$
\tilde{\mathbf{K}}=\left(\begin{array}{ccc}
r f & s & 0 \\
0 & f & 0 \\
0 & 0 & 1
\end{array}\right)
$$

and thus the matrix $\tilde{\mathbf{K}}^{-T} \tilde{\mathbf{K}}^{-1}$ can be expressed as

$$
\tilde{\mathbf{K}}^{-T} \tilde{\mathbf{K}}^{-1}=\left(\begin{array}{ccc}
k_{1} & k_{2} / 2 & 0 \\
k_{2} / 2 & k_{3} & 0 \\
0 & 0 & 1
\end{array}\right)
$$

where $k_{1}=\frac{1}{r^{2} f^{2}}, k_{2}=-\frac{2 s}{r^{2} f^{3}}, k_{3}=\frac{r^{2} f^{2}+s^{2}}{r^{2} f^{4}}$.

\subsection{Other Intrinsic Parameters and Mirror Parameter}

Equation (1) is equivalent to the following

$$
\lambda \mathbf{m}-\tilde{\mathbf{K}}(0,0, \xi)^{T}=\tilde{\mathbf{K}} \frac{\mathbf{R X}+\mathbf{t}}{\|\mathbf{R X}+\mathbf{t}\|} \approx \tilde{\mathbf{K}}(\mathbf{R}, \mathbf{t})\left(\begin{array}{c}
\mathbf{X} \\
1
\end{array}\right)
$$

Comparing (8) with the image formation of perspective camera, we find that $\lambda \mathbf{m}-\tilde{\mathbf{K}}(0,0, \xi)^{T}$ just can be regarded as an image point of $\mathbf{X}$ under a perspective camera, and it can be expressed as follows

$$
\lambda \tilde{\mathbf{K}}^{-1} \mathbf{m}-\left(\begin{array}{c}
0 \\
0 \\
\xi
\end{array}\right)=\left(\begin{array}{c}
u \\
v \\
1-\frac{\xi \mathbf{m}^{T} \tilde{\mathbf{K}}^{-T} \tilde{\mathbf{K}}^{-1} \mathbf{m}}{\xi+\sqrt{\xi^{2}+\left(1-\xi^{2}\right) \mathbf{m}^{T} \tilde{\mathbf{K}}^{-T} \tilde{\mathbf{K}}^{-1} \mathbf{m}}}
\end{array}\right)
$$

Let $\mu=\mathbf{m}^{T} \tilde{\mathbf{K}}^{-T} \tilde{\mathbf{K}}^{-1} \mathbf{m}-1=k_{1} u^{2}+k_{2} u v+k_{3} v^{2}$. Then the third component in equation (9) can be expressed by $\mu$ as $f(\mu)=$ $\frac{1-\xi \sqrt{1+\left(1-\xi^{2}\right) \mu}}{1-\xi^{2}}$. Denote $f_{l}(\mu)$ to be the $l$ th-order Taylor expansion of $f(\mu)$, then we have

$$
f_{l}(\mu)=\frac{1}{1+\xi}+\xi \sum_{n=1}^{l}(-1)^{n-1}\left(1-\xi^{2}\right)^{n-1} a_{n} \mu^{n}
$$

Multiplying $f_{l}(\mu)$ with $(1+\xi)$ and replacing $\mu$ with $k_{1} u^{2}+$ $k_{2} u v+k_{3} v^{2}$, we have

$$
\tilde{L}(u, v, l)=1+\sum_{p=1}^{l} \sum_{\substack{i+j=2 p, i, j \geq 0}} b_{i j} u^{i} v^{j}
$$

where $a_{p}=\prod_{i=1}^{p}(2 i-3) /\left(2^{p} p !\right)$

$$
\begin{aligned}
& b_{i j}=(-1)^{p-1} \xi(1+\xi)\left(1-\xi^{2}\right)^{p-1} a_{p} \\
& \sum_{\substack{m, n \geq 0, 2 m+n=i, m+n \leq p}} \frac{p !}{m ! n !(p-m-n) !} k_{1}^{m} k_{2}^{n} k_{3}^{p-m-n}
\end{aligned}
$$


the parameters $k_{1}, k_{2}, k_{3}$ in $b_{i j}$ are defined in equation (7), and they can be expressed by the catadioptric cameras.

The equation (11) gives the relationship between Taylor coefficients of $\tilde{L}(u, v, l)$ and catadioptric parameters, and we have the following equation by the coefficients of $\tilde{L}(u, v, 2)$

$$
\begin{aligned}
& b_{20}=-\frac{\xi(1+\xi)}{2} k_{1}, b_{11}=-\frac{\xi(1+\xi)}{2} k_{2} \\
& b_{02}=-\frac{\xi(1+\xi)}{2} k_{3}, b_{40}=\frac{\xi(1+\xi)\left(1-\xi^{2}\right)}{8} k_{1}^{2}
\end{aligned}
$$

And thus we get the following proposition .

Proposition 1. For a central catadioptric camera with $0<\xi \leq 1$, the following relations exist between the catadioptric parameters and the Taylor coefficients:

$$
\begin{aligned}
& \xi=\frac{b_{20}^{2}}{2 b_{40}+b_{20}^{2}}, f=2 \sqrt{\frac{k_{1}}{4 k_{1} k_{3}-k_{2}^{2}}} \\
& r=\frac{\sqrt{4 k_{1} k_{3}-k_{2}^{2}}}{2 k_{1}}, s=-\frac{k_{2}}{\sqrt{k_{1}\left(4 k_{1} k_{3}-k_{2}^{2}\right)}}
\end{aligned}
$$

where $k_{1}=-\frac{2 b_{20}}{\xi(1+\xi)}, k_{2}=-\frac{2 b_{11}}{\xi(1+\xi)}, k_{3}=-\frac{2 b_{02}}{\xi(1+\xi)}$.

Proof:

With $b_{20}$ and $b_{40}$ in (13), we can eliminate $k_{1}$ and get the mirror parameter $\xi$ expressed by $b_{20}$ and $b_{40}$ as follows:

$$
\frac{b_{20}^{2}}{2 b_{40}+b_{20}^{2}}=\frac{\frac{\xi^{2}(1+\xi)^{2}}{4} k_{1}^{2}}{2 \frac{\xi(1+\xi)\left(1-\xi^{2}\right)}{8} k_{1}^{2}+\frac{\xi^{2}(1+\xi)^{2}}{4} k_{1}^{2}}=\xi .
$$

Secondly, substitute the above formula of $\xi$ into (13), and we can express $k_{1}, k_{2}, k_{3}$ by $b_{20}$ and $b_{40}$ :

With $k_{1}, k_{2}$ in equation (8), we can get

$$
r^{2}=\frac{1}{k_{1} f^{2}}, \quad s=-\frac{k_{2}}{2 k_{1}} f .
$$

By substituting (15) into $k_{3}$ in equation (8), we have $k_{3}=\frac{k_{2}^{2}}{4 k_{1}}+$ $\frac{1}{f^{2}}$, and thus the focal length $f$ can be recovered as follows:

$$
f=2 \sqrt{\frac{k_{1}}{4 k_{1} k_{3}-k_{2}^{2}}} .
$$

Finally, the aspect ratio $r$ and skew factor $s$ can be recovered with the obtained with the focal length $f$ and (15).

Inspired by the formation of $\tilde{L}(u, v, l)$, we propose the following polynomial model for central catadioptric camera:

$$
L(u, v, l)=1+\sum_{p=1}^{l} \sum_{\substack{i+j=2 p \\ i, j \geq 0}} c_{i j} u^{i} v^{j}
$$

where $L(u, v, l)$ is called the $l$ th-order polynomial division model, and the coefficients $\mathbf{c}=\left(c_{i j}\right)$ are called the distortion parameters of $L(u, v, l)$.

Similar to equation (9), the homogenous coordinate of $(u, v)$ after rectification with the model $L(u, v, l)$ can be computed by

$$
\mathbf{m}_{u}=(u, v, L(u, v, l))^{T}
$$

For three image points $\left\{\mathbf{m}_{i}=\left(u_{i}, v_{i}, 1\right)^{T}\right\}_{i=1}^{3}$ of points on a $1 \mathrm{D}$ object, their corresponding rectified perspective image points can be computed by $\left\{\mathbf{m}_{u i}=\left(u_{i}, v_{i}, L\left(u_{i}, v_{i}, l\right)\right)^{T}\right\}_{i=1}^{3}$ as (18), and $\left\{\mathbf{m}_{u i}\right\}_{i=1}^{3}$ will be collinear. Thus the determinant of the three rectified points is zero

$$
\left|\begin{array}{ccc}
u_{1} & u_{2} & u_{2} \\
v_{1} & v_{2} & v_{2} \\
L\left(u_{1}, v_{1}, l\right) & L\left(u_{2}, v_{2}, l\right) & L\left(u_{3}, v_{3}, l\right)
\end{array}\right|=0
$$

Equation (19) can be written explicitly as a linear homogenous constraint on the distortion parameters $\mathbf{c}=\left(c_{i j}\right)$ in $L(u, v, l)$ as follows:

$$
\mathbf{D c}=0
$$

where $\mathbf{D}=\left(d_{1}, d_{2}, \ldots, d_{8}, d_{9}\right)$ and $\mathbf{c}=\left(c_{20}, c_{11}, c_{02}, c_{40}, c_{31}\right.$, $\left.c_{22}, c_{13}, c_{04}, 1\right)^{T}$.

For a triple image points, we can establish a set of homogeneous linear equations by piling up the vector $\mathbf{D}_{1}$, and then at least 8 constraints are sufficient for calibration. Thus, we use singular value decomposition to compute $\mathbf{c}=\left(c_{20}, c_{11}, c_{02}, c_{40}, c_{31}\right.$, $\left.c_{22}, c_{13}, c_{04}, 1\right)^{T}$. It can be proved that a $1 \mathrm{D}$ objects with four markers can provide two independent constraints (due to space limitation, the proof is omitted.), and the distortion parameters can be solved by at least four images of the 1D object at different positions.

In this work, we replace $b_{i j}$ in Proposition 1 with our estimated $c_{i j}$ to get the catadioptric parameters. The reason lies as follows: (1) We use the polynomial model $L(u, v, l)$ to approximate the true model $\tilde{L}(u, v, l)(l \rightarrow \infty)$ of central catadioptric camera, and the corresponding parameters in the two models should be close. (2) With the parameters $\left\{b_{i j}\right\}$, the ground truth intrinsic parameters of central catadioptric cameras can be computed with Proposition 1. In the experiments 4.1 , the calibrated parameter $c_{i j}$ in $L(u, v, l)$ is used to replace the parameter $b_{i j}$ in Proposition 1, and the estimated intrinsic parameters of the catadioptric cameras are close to the ground truth. Therefore, we can replace $b_{i j}$ in Proposition 1 with the estimated $c_{i j}$ to initialize the catadioptric parameters.

\subsection{Complete Algorithm}

An outline of our algorithm is summarized as follows: Input: images of 1D objects by at least five motions.

Output: parameters $f, r, s, u_{0}, v_{0}, \xi$.

1. Compute the principal point $\mathbf{p}=\left(u_{0}, v_{0}, 1\right)^{T}$ with the equations (4), and move the origin of image coordination to the principal point by (5);

2. Construct the linear constraints of parameters in the polynomial model by (20), and estimate the parameters with singular value decomposition;

3. The catadioptric parameters can be recovered with the obtained parameters in the polynomial model and Proposition 1;

4. Refine the catadioptric parameters by nonlinear optimization techniques, where the cost function is the sum of the residual squares of the equations (19).

\section{EXPERIMENTS}

We use simulated and real data to evaluate the performance of our method. In both simulated and real image experiments, we use the second order polynomial model $L(u, v, 2)$ for calibration. 


\subsection{Simulated experiments}

The intrinsic parameters of the simulated catadioptric cameras are $\left(r f, f, s, u_{0}, v_{0}\right)=(510,500,0,650,550)$. The mirror parameter is 0.9665 . The parameters keep unchanged when the $1 \mathrm{D}$ calibration object with five points is moving. The image resolution is of $1300 \times 1100$. We carry out simulated experiments under the parameter settings. Catadioptric images of 1D object are generated, and Gaussian noise with zero mean and standard deviation $\sigma=0,1, \ldots, 5$ pixels is added to the image points. For each noise level, we perform 50 trails. The relative errors of intrinsic parameters with respect to $f$, and the absolute error of $\xi$ are measured. The relative errors of the intrinsic parameters with only linear estimations are given in Fig. 2(a), and the absolute error of $\xi$ are given in Fig. 2(b). The errors of catadioptric parameters increase almost linearly as the noise level increases, and the errors are also fairly small.

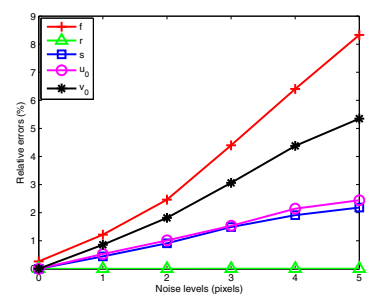

(a) (b)

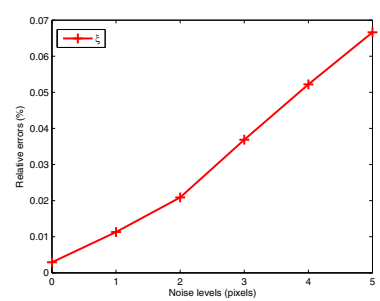

Fig. 2. The errors of catadioptric parameters. (a) the relative error of parameters $f, r, s, u_{0}, v_{0}$. (b) the error of parameter $\xi$.

\subsection{Real experiments}

The used central catadioptric camera is with a hyperboloid mirror designed by Czech Technical University, and the image resolution is $2048 \times 1536$ pixels. The $1 \mathrm{D}$ object is a stick with five markers, and each pairs of neighbor markers are $15 \mathrm{~cm}$ apart. The stick was moved 10 times, and one of the captured images are shown in Fig. 3(a). The images of five markers are selected manually and shown by red color. The calibration results by our method(1D) are shown in Tab. 1. We use estimated field of view(FOV) and mirror parameter $\xi$ to evaluate the calibration results(See Tab. 1). The estimated FOV and $\xi$ by our method are fairly close to the ground truth 212 degrees and 0.9665 respectively, which are provided by the mirror manufacturer. Moreover, the rectified image with the calibration results by our method is shown in Fig.3(b), in which heavy distortions are removed. From the comparisons, we see our method is reliable.

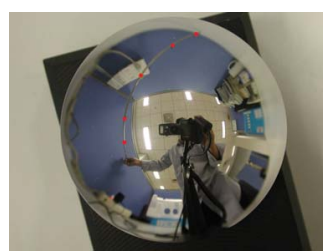

(a)

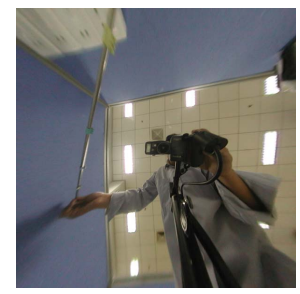

(b)
Fig. 3. Experiments using real images. (a) catadioptric image. (b) rectification with the results by our method.
Table 1. Calibration results by our method(1D).

\begin{tabular}{|c|c|c|c|c|c|c|c|}
\hline method & $f$ & $r$ & $s$ & $u_{0}$ & $v_{0}$ & $\xi$ & FOV \\
\hline 1D & 587.0 & 1.0 & -15.3 & 1046.1 & 759.9 & 1.0 & 214.9 \\
\hline
\end{tabular}

\section{CONCLUSION}

In this paper, a flexible $1 \mathrm{D}$ object based calibration method for catadioptric camera is proposed. Our method is capable of calibrating catadioptric camera with the 1D object undergoes five or more general motions. Our method has the advantages that no fitting of image points to conics and no prior knowledge to the catadioptric parameters are required. Experiments show our method is robust and reliable. Due to the flexibility of 1D object, our method can be also used in the calibration process of multiple catadioptric cameras.

\section{Acknowledgement}

This work is supported by National Natural Science Foundation of China under Grant No.61005039 and No.61075038, the open projects program of National Laboratory of Pattern Recognition of Institute of Automation, Chinese Academy of Sciences under Grant No. 20090096, and the Fundamental Research Funds for the Central Universities.

\section{REFERENCES}

[1] Baker S. and Nayar S., "A theory of single-viewpoint catadioptric image formation," IJCV, vol. 35, no. 2, pp. 175-196, 1999.

[2] Gioi R., Monasse P., Morel J., and Tang Z., “Towards highprecision lens distortion correction," ICIP, pp. 4237-4240, 2010.

[3] Deng X., Wu F., Wu Y., and Duan F., "Visual metrology with uncalibrated radial distorted images," ICPR, pp. 1-4, 2008.

[4] Wu Y., Li Y., and Hu Z., "Easy calibration for para-catadioptriclike camera," IROS, pp. 5719-5724, 2006.

[5] Mincuski B. and Pajdla T., "Structure from motion with wide circular field of view cameras," PAMI, vol. 28, no. 7, pp. 11351149, 2006.

[6] Gandhi T. and Trivedi M. M., "Reconfigurable omnidirectional camera array calibration with a linear moving object," IVC, pp. 935-948, 2006.

[7] Aliaga D.G., "Accurate catadioptric calibration for real-time pose estimation in room-size environments," in ICCV, 2001, pp. 127-134.

[8] Barreto J. P. and Araujo H., "Geometry properties of central catadioptric line images and application in calibration," PAMI, vol. 27, no. 8, pp. 1327-1333, 2005.

[9] Zhang Z., "Camera calibration with one-dimensional objects," PAMI, vol. 26, no. 7, pp. 892-899, 2004.

[10] Wang L., Wu F., and Hu Z., "Multi-camera calibration with one-dimensional object under general motions," ICCV, pp. 1-7, 2007.

[11] Geyer C. and Daniilidis K., "Catadioptric projective geometry," IJCV, vol. 43, pp. 223-243, 2001.

[12] Wu Y. and Hu Z., "Geometric invariants and applications under catadioptric camera model," ICCV, pp. 1547-1554, 2005. 